\title{
Summary of the In Vivo Predictive Dissolution (iPD) - Oral Drug Delivery (ODD) Conference 2018
}

Bart Hens ${ }^{1,2}$, Jozef Al-Gousous ${ }^{1}$, Kai Wang ${ }^{1}$, Niloufar Salehi ${ }^{3}$, Robert M. Ziff ${ }^{3}$, Yasuhiro Tsume ${ }^{1}$, Marival Bermejo ${ }^{1,4}$, Paulo Paixão ${ }^{1,5}$, James G. Brasseur ${ }^{6,7}$, Alex $\mathrm{Yu}^{1}{ }^{1}$, Arjang Talattof ${ }^{1}$, Gail Benninghoff ${ }^{1}$, Peter Langguth ${ }^{8}$, Hans Lennernäs ${ }^{9}$, William L. Hasler ${ }^{10}$, Luca Marciani ${ }^{11}$, Duxin Sun ${ }^{1}$, Gregory E. Amidon' ${ }^{1}$, Joseph Dickens ${ }^{12}$, Kerby Shedden ${ }^{12}$, Raimar Löbenberg ${ }^{13}$, and Gordon L. Amidon ${ }^{1 *}$

${ }^{1}$ Department of Pharmaceutical Sciences, College of Pharmacy, University of Michigan, Ann Arbor, MI, USA

${ }^{2}$ Department of Pharmaceutical and Pharmacological Sciences, Katholieke Universiteit Leuven, Leuven, Belgium

${ }^{3}$ Center for the Study of Complex Systems and Department of Chemical Engineering, University of Michigan, Ann Arbor, MI, USA

${ }^{4}$ Department Engineering Pharmacy Section, Miguel Hernandez University, Alicante, Spain

${ }^{5}$ Research Institute for Medicines (iMed.ULisboa), Faculty of Pharmacy, Universidade de Lisboa, Lisboa, Portugal

${ }^{6}$ Department of Mechanical and Nuclear Engineering, Pennsylvania State University, University Park, PA, USA

${ }^{7}$ Department of Aerospace Engineering Sciences, University of Colorado Boulder, Boulder, CO, USA

${ }^{8}$ Department of Pharmaceutical Technology and Biopharmaceutics, Johannes Gutenberg University Mainz, Mainz, Germany

${ }^{9}$ Department of Pharmacy, Uppsala University, Uppsala, Sweden

${ }^{10}$ Department of Internal Medicine, Division of Gastroenterology, University of Michigan, Ann Arbor, MI, USA

${ }^{11}$ Nottingham Digestive Diseases Centre and 2NIHR Nottingham Biomedical Research Centre at Nottingham University Hospitals NHS Trust and the University of Nottingham, Nottingham, United Kingdom

${ }^{12}$ Department of Statistics, University of Michigan, Ann Arbor, MI, USA

${ }^{13}$ Faculty of Pharmacy \& Pharmaceutical Sciences, University of Alberta, Edmonton, Alberta, Canada

D uring the in vivo predictive dissolution (iPD) - oral drug delivery (ODD) conference at Lake Tahoe from March 4-9, 2018, different speakers quoted the need of a biorelevant dissolution test to better reflect the intraluminal behavior of an oral drug product in the human gastrointestinal (GI) tract. All attendees agreed that replacement of human bioequivalence $(\mathrm{BE}) /$ bioavailability (BA) studies by a biorelevant dissolution test will result in a more rational selection of drug and formulation strategies in the pharmaceutical industry, resulting in time- and cost-effective research, which is a benefit for pharmaceutical companies and, in the end, patients. There is a tremendous need to create a more scientific framework to assess formulation performance with less ethical considerations supporting novel and generic drug product development. Invited guest speakers discussed their personal expertise in the field of oral drug product testing. The ongoing biopharmaceutical project OrBiTo (http://www.orbitoproject.eu) kicked off in 2012 in Europe to address the pharmaceutical companies' quest to improve the predictive power of in vitro dissolution methodologies. The structure and organization of the project was summarized by Hans Lennernäs. In addition, the first day discussed the different in vivo methodologies that can be applied to elucidate the behavior of on oral dosage form in the human GI tract with a specific focus on (i) aspiration/manometry studies (Gordon L. Amidon) and (ii) magnetic resonance imaging (MRI) studies
(Luca Marciani). In the end, the compatibility of using a customized catheter with an MRI device is interesting to simultaneously explore the volumes present in the GI tract and back-and-forth mixing of fluids during intraluminal profiling studies. Another option is to apply the wireless pharmaceutical analysis device (WiPAD) that is currently being tested in dogs as presented by Duxin Sun. Briefly, a GI sampling capsule has to be orally ingested together with a drug product of interest. During its residence time in the Gl tract, this capsule has the ability to take eight fluid samples at eight different regions inside the GI tract. After being emptied in the stool, the capsule can be recovered, opened, and the eight separated samples can be isolated and analyzed for drug content. This promising methodology is a less-invasive procedure compared to intubation studies, but development is still in the exploratory phase. The potential of applying positronemission tomography (PET) was explained by Shinji Yamashita, who compared concentrations of radiolabeled probes, e.g., $\left.{ }^{11} \mathrm{C}\right]$ telmisartan, in different tissues. This technique showed drug distribution of the radio-labeled drug along the $\mathrm{Gl}$ tract of a rat and human (1).

The second day of the conference focused on different physiological aspects of the Gl tract that should not be neglected when it comes to biorelevant dissolution testing. Different talks made the audience aware that (i) selection of the right buffer/buffer concentrations (Gordon

${ }^{*}$ Corresponding author 
L. Amidon and Gregory E. Amidon) and (ii) an adequate implementation of gastric motility and emptying ( Marival Bermejo and Paulo Paixão) are two critical parameters that should not be neglected if you want to draw the right conclusions related to drug product behavior in the human GI tract (2). The complexity of the stomach shows us that we would oversimplify the stomach whenever we would treat it as a single, well-mixed compartmental model. Based on intraluminal concentration-time profiles of the non-absorbable marker, phenol red, it became clear that the observed results along the $\mathrm{Gl}$ tract can only be explained if the stomach is handled as a multi-compartmental design, respecting the stomach's anatomy and physiology in fasted and fed states. Deanna Mudie (Lonza Pharma \& Biotech) summarized the important in vitro dissolution parameters that should be considered to set up a biorelevant dissolution test for poorly soluble drug compounds in industry based on dose number, dissolution number, and permeation number (3). Especially for predictions towards the intraluminal behavior of poorly soluble drugs with basic properties, a lot of in vitro work has been established throughout the years working with the GI simulator (GIS) and artificial stomach-duodenum (ASD) model, as shown by the last presentation of the day by Yasuhiro Tsume (4-6).

Day 3 of the conference presented academic and industry perspectives of performing biorelevant dissolution tests to select the right formulation(s) prior to the start of clinical trials. Ping Gao (AbbVie) and Deanna Mudie demonstrated the added value of a biphasic dissolution test (using an organic layer to mimic intestinal uptake of the drug) (7-9). Shinji Yamashita showed a quantitative assessment of oral drug absorption by working with an in vitro dissolution/permeation device (10). David C. Sperry (Eli Lilly and Company) demonstrated the practical use of combining biorelevant dissolution testing (performed in the ASD model) with physiologicallybased pharmacokinetic (PBPK) modeling to gain more information about drug product's behavior in humans $(11,12)$. Moreover, the application of PBPK modeling to look at drug-drug interactions (DDI) is becoming a popular strategy in a regulatory context; from July 2008 until June 2010, the United States Food and Drug Administration (FDA) reviewed seven investigational new drug (IND) products and six new drug products applications (NDA). Before 2008, the FDA received only two submissions containing PBPK modeling (13). Many of the PBPK modeling and simulation evaluations were addressing questions related to DDI and specific populations (e.g., pediatrics), which were described by Jianghong Fan of the FDA. From 2008 until 2015, the FDA received a total of 54 in vivo-in vitro correlations (IVIVC) submissions for NDA/IND approval; from the 54 submissions, 36 regulatory submissions containing IVIVC approaches were not accepted and, consequently, failed approval. The development and incorporation of biopredictive dissolution methods should be explored to increase the likelihood of IVIVC success (14).

On the fourth day, Raimar Löbenberg demonstrated how we should shift toward a more mechanistical product development with a scientific framework that supports IVIVC for different kinds oral drug formulations. He highlighted the benefit of coupling biorelevant dissolution testing with PBPK modeling to simulate the systemic outcome of oral drug products and to evaluate $\mathrm{BE}$ between tested and reference/marketed drug products. Greg Amidon, Niloufar Salehi, and James G. Brasseur showed us the impact of hydrodynamic enhancements on the dissolution rate of a drug. The application of computational fluid dynamics (CFD) for different in vitro models was illustrated and compared with in vivo shear rates (i.e., the rate at which a progressive shearing deformation is applied to some material) (15). This work clearly demonstrates the relevance of using the right dissolution vessel and impeller to create biorelevant conditions in terms of hydrodynamics. In addition to this work, a mass transport model (MTM) based on the design of the GIS was developed to consider shear rates in different Gl compartments. This model is a work in progress that, besides shear rates, also includes other physiological variables such as buffer properties, gastric emptying, pH, and secretions, as presented by Yasuhiro Tsume. The sensitivity of the integrated parameters can be essential to address potential BE failures whenever the marketed and tested drug product will be explored in the in vitro and in silico GIS device. This model expands the horizons to develop a scientific framework to evaluate IVIVC/IVIVR. Jack Cook (Pfizer) presented a survey that was conducted among 13 pharmaceutical companies (industry partners of the European IMI-funded project OrBiTo) to ask them about the current status of IVIVC development in pharmaceutical industry. The survey highlighted a lack of experience with respect to safespace in vivo-in vitro relationships (IVIVR) as well as the use of PBPK in the field of IVIVC. At the same time, the responses from both industry and regulatory agencies indicated that there might be a need for regulatory framework to guide the application of these novel approaches (16). The conclusion of this survey clearly emphasized that pursuing IVIVC should be generally encouraged, considering its high value from both industry and regulators' perspective. Both industry and

Dissolution 
regulatory authorities will benefit from a standardized biopredictive dissolution model that can establish IVIVC/IVIVR for different kinds of oral drug products. Regarding disintegration aspects for oral drug products, Peter Langguth demonstrated some case examples to show the audience that (i) the current pharmacopoeial disintegration tester can be modified for a better in vivo prediction and (ii) biopredictive disintegration methods in combination with dissolution data may help explain unexpected in vivo pharmacokinetic findings, such as food effects and effects of tablet crushing on dissolution of the drug.

The last day of the conference highlighted the evolution of generic drug development from the 1970s until now. Lawrence Yu brought to our attention how BE studies are evolving from an arbitrary to a more statistical design. A comparison was established between average BE criteria, using $80-125 \%$ limits on the $90 \%$ confidence intervals for Cmax and AUC geometric mean ratios, and a scaled average BE (i.e., tightened BE limits including withinsubject variability). Especially in the case of highly variable drug products (within-subject variability with $\mathrm{CV} \geq 30 \%$ ) and narrow therapeutic drug products, a scaled average $\mathrm{BE}$ is a good statistical and rational approach for the evaluation of the BE (17-20). In case of modified release dosage forms, multiphasic PK behavior (showing more than one peak/shoulder in the concentration profile) may be seen. If so, the traditional metrics of AUC and Cmax may not be sufficient to ensure $B E$. In addition to the traditional PK parameters of AUCinf and Cmax, partial AUC (pAUC; $A \cup C_{0-1.5 h}$ and $A U C_{1.5-t}$ ) measurements are recommended to provide $B E$ measures with respect to label indications (21). This was thoroughly explored and discussed for zolpidem tartrate extended-release tablets (Ambien $\mathrm{CR}^{\circledR}$ ) with an immediate-release layer on the outside of the tablet and a controlled-release layer on the inside. The rationale behind this formulation strategy is to launch a direct sleep onset followed by a sustained period for sleep maintenance. Regarding BE for delayed-release drug products, Peter Langguth and Jozef Al-Gousous observed that enteric-coated dosage forms frequently do not rapidly dissolve following gastric emptying and, thus, the pharmacokinetic lag-time does not only reflect the gastric emptying process (22).

\section{ACKNOWLEDGEMENTS}

This report represents the scientific views of the authors and not necessarily that of the FDA.

More information about the project and the iPD conference can be found on: http://www.ddfint.org/odd2018/

\section{CONFLICT OF INTEREST}

The authors disclosed no conflicts of interest related to this article.

\section{FUNDING}

This work was supported by grant no. HHSF223201510157C and grant no. HHSF223201310144C by the US Food and Drug Administration. Bart Hens received support from Internal Funds of KU Leuven (KU Leuven - Internal Funds PDM/17/164).

\section{REFERENCES}

1. Yamashita, S.; Takashima, T.; Kataoka, M.; Oh, H.; Sakuma, S.; Takahashi, M.; Suzuki, N.; Hayashinaka, E.; Wada, Y.; Cui, Y.; Watanabe, Y. PET imaging of the gastrointestinal absorption of orally administered drugs in conscious and anesthetized rats. J. Nucl. Med. 2011, 52, 249-256. DOI: 10.2967/ jnumed.110.081539.

2. Al-Gousous, J.; Amidon, G. L.; Langguth, P. Toward biopredictive dissolution for enteric coated dosage forms. Mol. Pharm. 2016, 13, 1927-1936. DOI: 10.1021/acs.molpharmaceut.6b00077.

3. Mudie, D. M.; Amidon, G. L.; Amidon, G. E. Physiological parameters for oral delivery and in vitro testing. Mol. Pharm. 2010, 7, 1388-1405. DOI: 10.1021/mp100149j.

4. Tsume, Y.; Igawa, N.; Drelich, A. J.; Amidon, G. E.; Amidon, G. L. The combination of GIS and biphasic to better predict in vivo dissolution of BCS class IIb drugs, ketoconazole and raloxifene. J. Pharm. Sci. 2018, 107, 307-316. DOI: 10.1016/j. xphs.2017.09.002.

5. Tsume, Y.; Takeuchi, S.; Matsui, K.; Amidon, G. E.; Amidon, G. L. In vitro dissolution methodology, mini-gastrointestinal simulator (MGIS), Predicts better in vivo dissolution of a weak base drug, dasatinib. Eur. J. Pharm. Sci. 2015, 76, 203-212. DOI: 10.1016/j. ejps.2015.05.013.

6. Carino, S. R.; Sperry, D. C.; Hawley, M. Relative bioavailability estimation of carbamazepine crystal forms using an artificial stomach-duodenum model. J. Pharm. Sci. 2006, 95, 116-125. DOI: 10.1002/jps.20495.

7. Gao, P.; Shi, Y. Characterization of supersaturatable formulations for improved absorption of poorly soluble drugs. AAPS J. 2012, 14, 703-713. DOI: 10.1208/s12248-012-9389-7.

8. Shi, Y.; Erickson, B.; Jayasankar, A.; Lu, L.; Marsh, K.; Menon, R.; Gao, P. Assessing supersaturation and its impact on in vivo bioavailability of a low-solubility compound ABT-072 with a dual pH, two-phase dissolution method. J. Pharm. Sci. 2016, 105, 2886-2895. DOI: 10.1016/j.xphs.2016.04.036.

9. Mudie, D. M.; Shi, Y.; Ping, H.; Gao, P.; Amidon, G. L.; Amidon, G. E. Mechanistic analysis of solute transport in an in vitro 
physiological two-phase dissolution apparatus. Biopharm. Drug Dispos. 2012, 33, 378-402. DOI: 10.1002/bdd.1803.

10. Kataoka, M.; Masaoka, Y.; Yamazaki, Y.; Sakane, T.; Sezaki, H.; Yamashita, S. In vitro system to evaluate oral absorption of poorly water-soluble drugs: simultaneous analysis on dissolution and permeation of drugs. Pharm. Res. 2003, 20, 1674-1680. DOI: 10.1023/A:10261079.

11. Ding, X.; Day, J. S.; Sperry, D. C. Physiologically based absorption modeling to design extended-release clinical products for an ester prodrug. AAPS J. 2016, 18, 1424-1438. DOI: 10.1208/ s12248-016-9950-x.

12. Polster, C. S.; Wu, S.-J.; Gueorguieva, I.; Sperry, D. C. Mechanism for enhanced absorption of a solid dispersion formulation of LY2300559 using the artificial stomach duodenum model. Mol. Pharm. 2015, 12, 1131-1140. DOI: 10.1021/mp5006036.

13. Zhao, P.; Zhang, L.; Grillo, J. A.; Liu, Q.; Bullock, J. M.; Moon, Y. J.; Song, P.; Brar, S. S.; Madabushi, R.; Wu, T. C.; Booth, B. P.; Rahman, N. A.; Reynolds, K. S.; Gil Berglund, E.; Lesko, L. J.; Huang, S. M. Applications of physiologically based pharmacokinetic (PBPK) modeling and simulation during regulatory review. Clin. Pharmacol. Ther. 2011, 89, 259-267. DOI: 10.1038/ clpt.2010.298.

14. Suarez-Sharp, S.; Li, M.; Duan, J.; Shah, H.; Seo, P. Regulatory Experience with in vivo in vitro correlations (IVIVC) in new drug applications. AAPS J. 2016, 18, 1379-1390. DOI: 10.1208/ s12248-016-9966-2.

15. Abrahamsson, B.; Pal, A.; Sjöberg, M.; Carlsson, M.; Laurell, E.; Brasseur, J. G. A novel in vitro and numerical analysis of shearinduced drug release from extended-release tablets in the fed stomach. Pharm. Res. 2005, 22, 1215-1226. DOI: 10.1007/ s11095-005-5272-x.

16. Nguyen, M. A.; Flanagan, T.; Brewster, M.; Kesisoglou, F.; Beato, S.; Biewenga, J.; Crison, J.; Holm, R.; Li, R.; Mannaert, E.; McAllister, M.; Mueller-Zsigmondy, M.; Muenster, U.; Ojala, K.; Page, S.; Parr, A.; Rossenu, S.; Timmins, P.; Van Peer,
A.; Vermeulen, A.; Langguth, P. A survey on IVIVC/IVIVR development in the pharmaceutical industry - past experience and current perspectives. Eur. J. Pharm. Sci. 2017, 102, 1-13. DOI: 10.1016/j.ejps.2017.02.029.

17. Davit, B. M.; Conner, D. P.; Fabian-Fritsch, B.; Haidar, S. H.; Jiang, X.; Patel, D. T.; Seo, P. R. H.; Suh, K.; Thompson, C. L.; Yu, L. X. Highly variable drugs: observations from bioequivalence data submitted to the FDA for new generic drug applications. AAPS J. 2008, 10, 148-156. DOI: 10.1208/s12248-008-9015-x.

18. Haidar, S. H.; Makhlouf, F.; Schuirmann, D. J.; Hyslop, T.; Davit, B.; Conner, D.; Yu, L. X. Evaluation of a scaling approach for the bioequivalence of highly variable drugs. AAPS J. 2008, 10, 450454. DOI: 10.1208/s12248-008-9053-4.

19. Yu, L. X.; Jiang, W.; Zhang, X.; Lionberger, R.; Makhlouf, F.; Schuirmann, D. J.; Muldowney, L.; Chen, M.-L.; Davit, B.; Conner, D.; Woodcock, J. Novel bioequivalence approach for narrow therapeutic index drugs. Clin. Pharmacol. Ther. 2015, 97, 286291. DOI: 10.1002/cpt.28.

20. Jiang, W.; Makhlouf, F.; Schuirmann, D. J.; Zhang, X.; Zheng, N.; Conner, D.; Yu, L. X.; Lionberger, R. A bioequivalence approach for generic narrow therapeutic index drugs: evaluation of the reference-scaled approach and variability comparison criterion. AAPS J. 2015, 17, 891-901. DOI: 10.1208/s12248-015-9753-5.

21. Lionberger, R. A.; Raw, A. S.; Kim, S. H.; Zhang, X.; Yu, L. X. Use of partial AUC to demonstrate bioequivalence of zolpidem tartrate extended release formulations. Pharm. Res. 2012, 29, 11101120. DOI: 10.1007/s11095-011-0662-8.

22. Al-Gousous, J.; Tsume, Y.; Fu, M.; Salem, I. I.; Langguth, P. Unpredictable performance of $\mathrm{pH}$-dependent coatings accentuates the need for improved predictive in vitro test systems. Mol. Pharm. 2017, 14, 4209-4219. DOI: 10.1021/acs. molpharmaceut.6b00877. 